\title{
BioChangeFields - vegetation database of arable plant communties in Central Germany
}

\author{
Stefan Meyer, Benjamin Krause \& Karsten Wesche
}

\begin{abstract}
The database BioChangeFields (GIVD ID EU-DE-027) aims at a comparison of arable plant vegetation in the 1950s/1960s and today. Historical vegetation samples were collected in 10 regions of Central and Northern Germany; three on loamy sites $(n=$ $122)$, four on sandy sites $(n=154)$ and three on calcareous sites (116 relevés). New vegetation data were collected by $S$. Meyer, B. Krause and K. Wesche. Usually, ca. 40 historical (field interior), ca. 40 recent samples (field interior) and another ca. 40 recent samples (field margin) are available, taken at approximately the same location.
\end{abstract}

Keywords: arable plant; Central Germany; re-sampling; segetal vegetation; Stellarietea mediae.

GIVD Database ID: EU-DE-027

Last update: 2012-05-01

\section{BioChangeFields}

Scope: Re-sampling of semi-permanent plots on arable fields (1950s/60s-2009) to analyse changes in species composition.

Status: completed and continuing

Period: 1951-2009

Database manager(s): Stefan Meyer (smeyer1@gwdg.de)

Owner: University of Goettingen

Dept. of Plant Ecology and Ecosystems Research

Web address: $[\mathrm{NA}]$

Availability: free upon request

Database format(s): TURBOVEG

Online upload: no

Online search: no

Publication: in progress

Plot type(s): normal plots; time series

Export format(s): TURBOVEG, MS Access, SQL, Excel, Open

Plot type(s): normal plots; time

Document, PDF

Total plot observations: 1,176

Plot-size range: $10-100 \mathrm{~m}^{2}$

Countries: DE: $100.0 \%$

Estimate of existing plots: [NA]

Number of sources: 0

Completeness: [NA]

Valid taxa: $[N A]$

Forest: $0 \%$ — Non-forest: aquatic: $0 \%$; semi-aquatic: 0\%; arctic-alpine: $0 \%$; natural: $0 \%$; semi-natural: $0 \%$; anthropogenic: $100 \%$

Guilds: all vascular plants: $100 \%$

Environmental data: altitude: $100 \%$; slope aspect: $100 \%$; slope inclination: $100 \%$; microrelief: $100 \%$; other soil attributes: $100 \%$

Performance measure(s): cover: $100 \%$

Geographic localisation: GPS coordinates (precision $25 \mathrm{~m}$ or less): 50\%; point coordinates less precise than GPS, up to $1 \mathrm{~km}$ : $50 \%$

Sampling periods: $1950-1959: 30.0 \%$; 1960-1969: 20.0\%; 2000-2009: 50.0\%

Information as of 2012-07-12; further details and future updates available from http://www.givd.info/ID/EU-DE-027

Stefan Meyer* (smeyer1@gwdg.de), Benjamin Krause (bkrause@gwdg.de)

Department of Plant Ecology and Ecosystems Research, University of Goettingen, Untere Karspüle 2, 37073 Göttingen, GERMANY

Karsten Wesche (karsten.wesche@ senckenberg.de)

Department of Botany, Senckenberg Museum of Natural History, Am Museum 1, 02826 Görlitz, GERMANY

*Corresponding author 